\title{
Los MOOC en la formación continua y especializada: ¿nuevas narrativas y formatos audiovisuales? Mitos y retos
}

\section{(The MOOC in Continuous and Specialized Training: New Narratives and Audiovisual Formats? Myths and Challenges)}

\author{
Raquel Caerols Mateo \\ Universidad Complutense de Madrid (España) \\ Pavel Sidorenko Bautista \\ Universidad Francisco de Vitoria (España) \\ Sara Osuna-Acedo \\ Universidad Nacional de Educación a Distancia, UNED (España)
}

DOI: https://doi.org/10.5944/ried.25.1.30023

\section{Cómo referenciar este artículo:}

Caerols Mateo, R., Sidorenko Bautista, P., y Osuna-Acedo, S. (2022). Los $\mathrm{MOOC}$ en la formación continua y especializada: ¿nuevas narrativas y formatos audiovisuales? Mitos y retos. RIED. Revista Iberoamericana de Educación a Distancia, 25(1), pp. 81-99. https://doi.org/10.5944/ried.25.1.30023

\section{Resumen}

Los formatos audiovisuales educativos tienen su propia unicidad en relación con sus estructuras y narrativas, suponiendo una vuelta de tuerca más, una especialización de los mismos en lo referente al contexto digital. En ese sentido, el audiovisual, el vídeo en sí, son piezas centrales de los llamados MOOC, formatos de aprendizaje y formación nacidos al albor del propio espacio digital. El presente estudio, pretende abordar estas cuestiones desde la fundamentación teórica de la Educomunicación contemporánea y sus propuestas del transliteracy, pues entendemos que esta área de conocimiento es la idónea, ya que las bases de su constructo teórico se formularon sobre la relación medios de comunicación y educación. Sumando a ello, realizaremos una revisión de la literatura sobre el surgimiento de los MOOC y la era postMOOC, con el objetivo de evidenciar que la naturaleza de los citados MOOC, como su especificidad para la formación continua y especializada, así como de los medios audiovisuales que los componen, deben construir sus propias metodologías y narrativas. Para ello, aplicaremos plantillas de análisis de dichas piezas audiovisuales que nos llevarán a valorar en qué medida estas premisas metodológicas son una realidad o se siguen 
manteniendo patrones tradicionales, con la intención final de ser propositivos en líneas futuras de actuación.

Palabras clave: educación tecnológica; nuevas tecnologías; formación continua; aprendizaje asistido por ordenador; uso didáctico del ordenador.

\begin{abstract}
Educational audio-visual formats have their own uniqueness in relation to their structures and narratives, which is another turn of the screw, a specialization of them in relation to the digital context. In this sense, the audiovisual, the video itself, are central pieces of the socalled MOOC, learning and training formats born at the dawn of digital space itself. This study aims to address these issues from the theoretical foundation of contemporary education and communication and its proposals of transliteracy, as we understand that this area of knowledge is ideal, since the basis of its theoretical construct was formulated on the relationship between media and education. In addition, we will review the literature on the emergence of the MOOC, with the aim of highlighting that the nature of the MOOC and the postMOOC era, as its specificity for continuous and specialized education, as well as the audiovisual media that compose them, must build their own methodologies and narratives. For this purpose, we will apply analysis templates of these audiovisual pieces that will lead us to assess to what extent these methodological premises are a reality or still maintain traditional patterns, with the ultimate intention of being proactive in future lines of action.
\end{abstract}

Keywords: technologic education; new technologies; continuous training; computer aided learning; didactic use of the computer.

Si hay un campo de conocimiento que puede dar respuesta al papel que pueden y deben jugar los medios de comunicación en su relación con la educación y, más concretamente con el contexto actual, esa es la Educomunicación y, específicamente para los intereses de la presente investigación, el de los MOOC.

La 'Televisión Educativa' fue el primer formato con entidad propia que supuso un uso de la tecnología para el diseño de contenidos audiovisuales educativos. Uno de sus objetivos principales fue tratar de acercar la educación a poblaciones físicamente dispersas, sobre todo de zonas con muy escasos recursos económicos, en los que no se podían desarrollar los sistemas presenciales de educación. Un ejemplo destacado corresponde a la Telesencundaria en México (1968), con el objetivo de llevar la educación secundaria a las zonas rurales. Esta iniciativa fue reforzada en 1995 con Edusat, que empezó a transmitir vía satélite.

Curiosamente, tomando como referencia estos comienzos de la Televisión Educativa parece que, por una extraordinaria situación de pandemia como la que corresponde a la COVID-19, cobran verdadero sentido, pues se trata de un contexto en el que no ha habido otra vía de llevar la educación a cualquier lugar del mundo 
que los medios audiovisuales, ahora mediante recursos digitales o donde estos aún no llegan, el propio medio televisivo, o incluso la radio como herramienta educativa (Makazaga, 2020).

Como ya señaló la Educomunicación desde sus inicios, la tecnología es un medio o, como afirma Daniel Prieto Castillo (citado en Aparici, 2016) en la siguiente entrevista: http://bit.ly/385boVX, se trata de una herramienta más de mediación.

$\mathrm{Si}$ algo ha dejado en evidencia el contexto de pandemia ya referido, son dos cuestiones fundamentales: el valor de la presencialidad y el hecho de que las tecnologías necesitan sus propias metodologías y pedagogías. En esa dirección se puede afirmar que es imposible hablar de educación online en sí, pues esta se fundamenta en requerimientos de otra naturaleza, como bien afirmó Daniel Prieto Castillo (2004, p. 13) en su texto La comunicación en educación: "el hecho educativo es profunda, esencialmente comunicacional. La relación pedagógica es en su fundamento una relación entre seres que se comunican, que interactúan, que se construyen en la interlocución".

Es preciso plantear la razón por la cual los instrumentos educativos MOOC no son sustitutivos de los contextos de formación y educación presencial, sino complementarios, que se nutren en su retroalimentación y que poseen otras potencialidades de formación y aprendizaje a partir de otras fórmulas comunicacionales.

Por tanto, dicho esto, el objetivo fundamental que planteamos es, hasta qué punto los MOOC de las universidades más prestigiosas y los más demandados, asumen estos retos o si, por el contrario, las narrativas, los formatos audiovisuales y sus metodologías están aún muy lejos de responder al nuevo modelo formativo del espacio red.

Asimismo, resulta por igual necesario evidenciar que son formatos cuyo valor añadido radica en la oferta de otras posibilidades como la formación continua y especializada y, la creación de redes y comunidades de aprendizaje, en cuyos casos se requiere de metodologías específicas y dedicadas, ¿̇está sucediendo así?

\section{Los MOOC en el contexto de la educomunicación y del transliteracy: sus potencialidades en el espacio digital}

Los MOOC tienen su verdadera significación en el contexto digital, es decir, es consustancial a dicho contexto y ello supone que, conllevan una reformulación de los métodos y vías de aprendizaje. Los MOOC no pueden ni deben ser entendidos como alternativa y sustitución de los modelos presenciales de educación en la formación de la ciudadanía, pero sí deben procurar nuevos espacios educativos, flexibles y específicos (King et al., 2018).

En definitiva, deben sumarse como variables de este proceso, las transformaciones que proponen las nuevas generaciones con la incursión de las tecnologías en sus 
vidas y, como no, en las aulas, como es el caso actual de la 'Generación Z' (Baysal, 2014; Turner, 2015).

En ese sentido, el mejor ámbito de estudio para un análisis sólido y con sentido para el estudio propuesto es el de la Educomunicación, cuyo origen remite a los medios masivos en la sociedad en los años 50-60 del pasado siglo. En aquel momento empezaba a ser necesaria una mirada crítica hacia ellos, lo que dejaba en evidencia un abordaje imprescindible desde la educación, el papel que podían y debían ocupar en los procesos de aprendizaje, así como en el propio desarrollo de las metodologías empleadas en el ámbito educativo. Lo mismo vuelve a ocurrir en el contexto de las tecnologías digitales, lo que pareciera denotar un eventual regreso a errores presumiblemente de ese período pasado.

Desde la llegada de los medios masivos al ámbito educativo en el contexto analógico, es decir, prensa, radio, televisión y cine hasta las tecnologías digitales, el marco teórico y los lineamientos de la Educomunicación se han ido transformando significativamente.

Desde los primeros trabajos de los propios teóricos, investigadores y estudiosos de la materia (Paulo Freire, Mario Kaplún, Daniel Prieto Castillo, Francisco Gutiérrez o Len Masterman), así como desde las propias instituciones que abordaron el nuevo reto contemporáneo, como el caso de la UNESCO por ejemplo, que desarrollan sus estudios y debates en el marco de lo que se definió como alfabetización mediática o medialiteracy ${ }^{1}$, ha habido un largo recorrido de publicaciones científicas (como alguna ya citada e iremos citando a lo largo del texto por parte de los padres de la Educomunicación), reuniones y encuentros internacionales ${ }^{2}$, hasta la reformulación de las concepciones primigenias de la materia, llevando a plantear nuevos conceptos como el de las alfabetizaciones múltiples o transliteracy (Sukovic, 2016; Wilkinson, 2011).

Este último concepto se refiere a la habilidad para leer, escribir e interactuar en un amplio rango de plataformas, herramientas y medios, desde el canto a la oralidad, desde los manuscritos impresos, los media, hasta las redes sociales (Ipri, 2010). Para Andretta (2009) se trata de flexibilidad, adaptación y cambio constante en aras de la innovación mediante la creatividad con base en múltiples plataformas, y mediante mensajes lineales como no lineales, es decir, la misma narrativa y estructura de los MOOC. En otras palabras, la clave está en la idea de aprendizaje y creación colectiva frente a lo individual (Suárez, 2010, p. 35).

En cuatro décadas se ha pasado rápidamente de la simple gestión digital de contenidos hasta la formación en abierto propuesta por los MOOC (García-Peñalvo et al., 2017, p. 119).

Para profundizar aún más al respecto, el texto Alfabetización mediática e informacional: curriculum para profesores de UNESCO (2011), plantea la alfabetización mediática desde una convergencia de la radio, televisión, Internet, periódicos, libros, archivos digitales y bibliotecas a partir de una sola plataforma y de manera holística. 
Otros manuales como Education \& Training, referido a la Cooperación de la Unión Europea en el ámbito de la educación y la formación (ET 2020), el G2O Leaders' declaration Building consensus for fair and sustainable development, la Education Policy Outlook 2018 (publicado por la OECD) y al texto 2021 Metas Educativas. La educación que queremos para la generación de los bicentenarios, se centra por igual en garantizar una educación inclusiva y equitativa de calidad, al tiempo de promover oportunidades de aprendizaje permanente, pedagogías y métodos innovadores, así como habilidades digitales, en consonancia con los preceptos de le Educomunicación contemporánea. Esto permite introducir en el aprendizaje a lo largo de la vida o lifelong learning, donde los MOOC también encuentran su razón de ser en la formación continua y especializada.

De aquí surgen conceptos alternativos como edupunk ideado ya en 2008 por Jim Groom, referido a que cada individuo cree su propio proceso de aprendizaje, o el concepto do-it-yourself (hazlo tú mismo). Así han reflexionado autores como Howard Rheingold (2004) o Pierre Levy (2004) sobre la inteligencia colectiva, sobre las inteligencias múltiples, la cosmopedia o el supralenguaje, para definir las potencialidades de las tecnologías contemporáneas (Barbas, 2012). Es decir, es un cambio de paradigma.

Al hablar de hipermediación se hace referencia a procesos de intercambio, producción y consumo simbólico desarrollados en un entorno determinado por una gran cantidad de sujetos, medios y lenguajes interconectados tecnológicamente de manera reticular entre sí. Se trata específicamente de la trama de reenvíos, hibridaciones y contaminaciones que la tecnología digital procura por igual en el ecosistema mediático (Scolari, 2008, pp. 113-114).

Se trata de prácticas hipermediáticas que propician modelos de comunicación y aprendizaje rizomáticos e interactivos. Los planteamientos de la Educomunicación, de las llamadas alfabetizaciones múltiples, ya trabajan desde estos lineamientos y, sin duda, apuntan a la necesaria transformación de los modelos educativos y de aprendizaje. Por tanto, los MOOC son una respuesta natural y necesaria a este contexto, se explican desde él.

Así, se puede afirmar que los principios de la Educomunicación contemporánea, el marco teórico de las alfabetizaciones múltiples o el trasliteracy, es el enfoque desde el que deben ser concebidos los MOOC, pues cobran más sentido. Hay que tomar conciencia de que este debe ser su marco de referencia para que tengan un sentido como un modelo de aprendizaje propio pues, precisamente, son un modelo de aprendizaje específico, que no se puede equiparar ni sustituir a otros, sino retroalimentar. Y desde esta perspectiva también se evidencia que uno de sus sentidos y potencialidades fundamentales es el de la formación continua y especializada, como veremos en los análisis posteriores. 


\section{Los MOOC como herramienta educativa en el contexto digital: plataformas, formatos, estructuras narrativas y sus metodologías}

Antes de la puesta en marcha de los primeros MOOC y plataformas principales para los mismos, surgieron diferentes iniciativas, siendolos primeros el Massachusetts Institute of Technology (MIT), publicando cursos de libre acceso en Internet a partir del 2002. No obstante, la fecha que se referencia en los textos especializados como punto de partida para el inicio formal de los MOOC es 2011, fue cuando los profesores de la Universidad de Stanford, Sebastian Thrun y Peter Norvig, lanzaron el primer MOOC de impacto internacional, dedicado a la inteligencia artificial.

El éxito en el número de matriculaciones (ciento sesenta mil personas de ciento noventa países), les llevó a fundar la plataforma Udacity (véase https://www.udacity. com/) en 2012 (Ruiz Bolívar, 2015, p. 7). No obstante, podemos afirmar que, entre el año 2011 y 2012 se crearon las principales plataformas para MOOC en el que estaban implicadas las principales universidades del mundo, de tal manera que:

- En 2011 se creó la plataforma Coursera (véase https://es.coursera.org/), por dos profesores de Ciencias de la Computación de la Universidad de Stanford (Andrew Ng y Daphne Koller), con cursos desarrollados en inglés, francés, español, italiano y chino.

- En 2012 también se fundó edX (véase https://www.edx.org/es), plataforma fundada por el MIT y la Universidad de Harvard.

- En noviembre del mismo año, 2012, se funda MiriadaX (véase https://www. miriadax.net/), plataforma que trabaja con el software libre WEMOOC, con el apoyo de Telefónica y Banco Santander, centrada en la formación online por parte de universidades iberoamericanas.

Asimismo, diferentes países como España, Irlanda, Reino Unido y Alemania han ido creado sus propias plataformas MOOC. En América Latina y Asia también es posible ver hoy una amplia oferta. En resumen, se puede destacar: Coursera, edX, Udemy, UNED, Miríada X, ECO-Learning, Tutellus, Mooc.es, INTEF, UPVX, Cript4you, Unimooc, Unx, Iversity, Acamica, Udacity, Khan Academy, Stanford MOOC, MIT OpenCourseware, CMU Open Learning Initiative, Open Learning, FutureLearn, Crehana, Codeacademy, Lynda, OpenHPI, XuetangX, Kadenze, Canvas Network, MexicoX, etc.

Del nacimiento de todas estas plataformas surgen dos grandes clasificaciones de MOOC: los cMOOCs y los xMOOCs. Los primeros responden a una estrategia conectivista (precursores Siemens y Downes, 2012), es decir: "se apoyan en un aprendizaje auto-organizado, centrado en obtener el significado de la experiencia de aprendizaje con la comunidad, para lo cual resultan claves las herramientas de participación como los blogs, feeds, RSS y otros métodos descentralizados" (Torres 
y Gago, 2014, p. 14). Los segundos se centran fundamentalmente en el contenido, en el sentido clásico del término con herramientas de clasificación sistematizadas.

Pero más allá de estos dos grandes grupos han surgido otras clasificaciones en la era postMOOC, como la Clark (2013), que distingue ocho tipos de MOOC, o la de Conole (2013), que los clasifica a partir de doce dimensiones como la capacidad de participación que se les permite a los participantes, tipo de aprendizaje, uso multimedia, entre otros. Por su parte, sMOOC y tMOOC modelos coherentes con los preceptos de la Educomunicación (Osuna-Acebo et al., 2018), se basan en las teorías conectivistas del aprendizaje, como los cMOOC, dando un paso más allá: el carácter social en la construcción del conocimiento, el trabajo colaborativo, la inclusión social y la conformación de comunidades virtuales, la intercreatividad y la transferencia inmediata de lo aprendido al ámbito social.

La aparición de la era postMOOC muestra la gran evolución y constante cambio del concepto, así como el replanteamiento de sus posibilidades y usos, pues cada vez se evidencia más su potencial y las múltiples posibilidades de combinación, es decir, se pueden diseñar a la carta en comunidades de interés de aprendizaje.

Ahora bien, esas múltiples posibilidades que apuestan por el aprendizaje, cómo se traducen en la realidad tangible de los MOOC más demandados y, a la vez permite preguntar, a qué modelos y metodologías de aprendizaje responden éstos en función de los modelos educativos que se establecen desde las ciencias de la educación.

Cabe reiterar que el punto de referencia teórico es la Educomunicación, pues desde este ámbito del saber, sus pioneros señalaron diferentes modelos educativos que pusieron en paralelo con la comunicación, para analizar y establecer también categorías para el estudio de la propia comunicación. Es decir, poner sobre la mesa y analizar los diferentes modelos educativos/comunicativos que aborda la literatura científica de la Educomunicación, nos servirá para saber en qué medida estos formatos son un aporte significativo según los modelos de aprendizaje de la Educomunicación, más allá de las tecnologías implicadas en el desarrollo de estos.

Así, en primer lugar, es necesario referir a uno de los padres de la Educomunicación: el pedagogo Paulo Freire (1968), en esta relación de interacción en la que se define la Educomunicación, su texto Pedagogía del oprimido establece ya unas claras conexiones entre ambas materias a partir de un análisis de los modelos de educación de su contemporaneidad que define, precisamente, como pedagogía del oprimido y que se relaciona directamente con el modelo de educación tradicional, al que denominará modelo bancario, describiéndolo como paternalista, vertical y autoritario, y que eso le llevará a una profunda reflexión, apelando a un necesario giro en estas metodologías educativas, y que deberían ir encaminadas hacia propuestas educativas participativas y dialógicas.

Mario Kaplún (1998) tomando como referencia e inspiración dicho texto, traslada éstas a su trabajo en el campo de la comunicación. Él establecerá dos clasificaciones de modelos educativos que encontrarán su paralelismo en el desarrollo de la labor 
comunicativa. Los modelos educativos que describirá son: el conductista (énfasis en los efectos) y el participativo (énfasis en el proceso).

Estos dos últimos los encuadra dentro de lo que denomina modelo endógeno, es decir, que se tiene en cuanto al sujeto, y el primero lo señala como modelo exógeno, en el que el sujeto queda fuera. Sin duda, la apuesta pedagógica de la Educomunicación será la de un modelo participativo (Osuna-Acedo, 2014). Es decir, al igual que la propuesta para el ámbito educativo se centró en una superación de los modelos de educación bancario y conductista, en el campo de la comunicación también será en un ir un más allá de producir y difundir, es decir, la intencionalidad fue poner en práctica y ejercer una comunicación que invitara al pensamiento crítico.

Estas referencias son idóneas para analizar qué lugar ocupan los MOOC y, concretamente, sus piezas audiovisuales, para poder hacer una valoración crítica de hasta qué punto las tipologías de material utilizado y las técnicas y estructuras narrativas de éstos, responden a las narrativas transmedia (Gil-Quintana y OsunaAcedo, 2020), es decir, a un espacio participativo, colaborativo o cocreativo. O, dicho de otro modo, si están dentro de un modelo bancario, tradicional (exógeno) o conductista o participativo (endógeno).

Ahora bien, al poner la mirada en teorías contemporáneas de la Educomunicación, como a las referidas anteriormente, transliteracy sí responde a prácticas transmedia innovadoras y creativas, sí requiere una participación, o acogen tanto mensajes lineales como no lineales (Gil-Quintana y Osuna-Acedo, 2020). Y, así mismo, permite afirmar que también responden a un modelo de edupunk ya señalado antes, referido a un modelo educativo que se define por crearse cada uno su propio proceso de aprendizaje.

Asimismo, se puede tomar como referencia de análisis para poner en valor su innovación en el contexto digital, las teorías de aprendizaje más influyentes desde los años 40 del siglo pasado hasta la actualidad, a partir de las cuales veremos la conexión directa con las establecidas por los padres de la Educomunicación, entendidas como: conductismo (una de las establecidas por Kaplún, 1998), cognitivimos y construcitivismo.

En las tres clasificaciones se habla de modelos endógenos, es decir, que tiene en cuenta al alumno/a, especialmente en el modelo cognitivista, pues pone énfasis en la participación del estudiante en su aprendizaje y el constructivista, para el que tiene especial importancia la interacción estudiante-profesor.

Los padres de la Educomunicación añaden un modelo que da un paso más allá y que, además, parece anticiparse a un modelo de aprendizaje que será el genuino del espacio digital y el entorno red, el participativo-cocreativo, en la misma medida que lo fue Toffler (1980) con la ideación del concepto prosumer, antes de que estuviera aquí internet. ¿En qué medida los MOOC y los materiales audiovisuales seleccionados responde a ese modelo?

Construida esta articulación teórica hay elementos teóricos que permiten aplicar las plantillas de análisis al material seleccionado, y así a través de estas premisas 
cualitativas, ver en qué medida responden a unos modelos de aprendizaje u otro y si responden a nuevas metodologías o narrativas propias del espacio red, si los materiales audiovisuales responden a ello y a los lineamientos del trasliteracy.

\section{METODOLOGÍA}

Para aportar propuestas, reflexiones, nuevas vías de trabajo e investigación y metodologías en el contexto de los MOOC y más concretamente, en las piezas audiovisuales que los componen, como elemento central de éstos, se plantea una metodología de análisis de los mismos, desde el papel que juegan en el contexto digital, poniendo como marco teórico de referencia el de la Educomunicación, por las cuestiones ya señaladas.

Una vez desarrollada la base teórica, se procederá a describir la estructura sobre la que se han ido construyendo los MOOC: plataformas y sus tipologías, tipos y formatos de los MOOC, metodologías sobre las que se sustentan, tipologías de piezas audiovisuales como herramientas centrales de los mismos en un contexto digital y sus estructuras narrativas. Ello permitirá plantear una plantilla de análisis que, principalmente, se centrará en las piezas audiovisuales de los MOOC seleccionados.

Para acotar la muestra se ha establecido como criterio de selección la plataforma digital 'edX', fundada por la Universidad de Harvard y el Massachusets Institute of Technology (MIT), la cual pugna con Coursera como líder mundial en oferta de cursos y programas de especialización en modalidad on line. Asimismo, ha sido seleccionada por provenir de entidades educativas referenciales.

Se trata de una plataforma del tipo XMOOx (constructivista) (Yousef et al., 2014) que aglutina ciento treinta y dos instituciones formativas y universidades de prestigio de todo el mundo, donde destacan los casos españoles de la Universidad Politécnica de Valencia, Universidad Autónoma de Madrid y Universidad Carlos III, concentra más de 2800 cursos que van desde las ciencias puras a las ciencias sociales y humanidades, y ostenta más de cien millones de inscripciones en cursos, los cuales son visualizados y discriminados en dos secciones: "Temas más populares” y “Todos los temas".

A diferencia del estudio propuesto por Rajas et al. (2018), para los fines específicos de esta investigación se trabajará con los programas más populares dentro del apartado "Temas más populares", entendiendo que se trata de los que gozan de mayor demanda por parte de los estudiantes.

El período de observación ha correspondido a la segunda quincena del mes de noviembre 2020 obteniendo como resultado diez cursos, dos por cada tema destacado, distribuidos de la siguiente manera (se presentan en el mismo orden de prioridad que asigna la plataforma): 
- Análisis de datos:

- Data Science, R Basics (Harvard https://bit.ly/3lVjYdW)

- Python Basics for Data Science (IBM https://bit.ly/35OQitq)

- Humanidades:

- IELTS Academic test preparation (University of Queenland https://bit. ly $/ 3 \mathrm{kNj} 5 \mathrm{mB}$ )

- Rhetoric, The art of persuasive writing and public speaking (Harvard https:// bit.ly/337BhBf)

- Idiomas:

- IELTS Academic test preparation (University of Queenland https://bit. ly $/ 3 \mathrm{kNj} 5 \mathrm{mB}$ )

- TOEFL test preparation, The insider guide (ETSx https://bit.ly/35OfujU)

- Informática:

- CS50's introduction to artificial intelligence with Python (Harvard https:// bit.ly/2UJBje6)

- Data Science, R basics (Harvard https://bit.ly/3lVjYdW)

- Negocios y administración:

- Entrepreneurship in emerging economies (Harvard https://bit.ly/3pPyre4)

- Contract law, from trust to promise to contract (Harvard https://bit. ly/3lRgPip)

La observación se ha centrado únicamente en el material audiovisual, obviando recursos de otro estilo como foros de discusión, guías de apoyo y evaluaciones.

Los datos obtenidos a partir de los criterios establecidos anteriormente conforman una tabla de análisis, la cual permite facilitar su visualización y posterior manejo de cara a la discusión en torno a ellos, tomando en cuenta como variables: Universidad o institución educativa, curso, número total de inscritos hasta el período de observación, total de recursos audiovisuales, promedio de tiempo total de todos los recursos audiovisuales, tipo de material audiovisual utilizado.

El material clasificado dentro de esta última variable estará identificado como: videoconferencia, vídeoclase y video apunte (VC-VP), reportaje-documental (RD), entrevista (E), debate, mesa redonda o tertulia (D-MR-T), dramatización (DR), ficcional (F), animación (AN), spot (S), contenido interactivo del tipo hipervídeo o colaborativo (INT).

Todas estas clasificaciones establecidas llevan implícitas también unas estructuras y técnicas narrativas, sobre las que se planteará el análisis correspondiente.

\section{RESULTADOS}

De la muestra señalada finalmente se revisaron ocho cursos pues dos se repiten en diferentes categorías como programas de formación muy demandados. Al hacer referencia a la Tabla 1, lo primero que vale señalar es que la Universidad de Harvard 
es la institución educativa que tiene el mayor número de cursos en diversos ámbitos, con los mayores índices de alumnos inscritos.

No obstante, queda también en evidencia que no tienen un modelo específico para elaborar este tipo de material educativo, y que el mismo se construye con unas particularidades narrativas dependiendo del tema que aborda. Así, como ocurre con los casos N. ${ }^{\circ}$ 1, 4, 6, 7 y 8 (Tabla 1) desarrollados por dicha universidad, destaca tanto la disparidad de tiempo total que acumula el contenido audiovisual, así como la cantidad de piezas específicamente.

Esta diferencia es más apreciable si se compara con el resto de los cursos presentados por otras instituciones. Tampoco pareciera haber una relación entre un período específico de contenidos y una mayor demanda del curso, todo lo contrario, resulta una variable determinada por el tema y seguramente por quien lo imparte, aunque la imagen institucional evidentemente tiene un peso por su prestigio y trayectoria.

\section{Tabla 1}

MOOC más populares en la plataforma 'edX' del 15 al 30 del noviembre 2020

\begin{tabular}{|c|c|c|c|c|c|c|}
\hline No & $\begin{array}{l}\text { Universidad } \\
\text { o institución } \\
\text { formativa }\end{array}$ & Curso & $\begin{array}{l}\text { Número } \\
\text { total de } \\
\text { inscritos }\end{array}$ & $\begin{array}{c}\text { Total de } \\
\text { recursos } \\
\text { audiovisuales }\end{array}$ & $\begin{array}{c}\text { Total de } \\
\text { tiempo de } \\
\text { los recursos } \\
\text { audiovisuales }\end{array}$ & $\begin{array}{c}\text { Tipo de } \\
\text { material } \\
\text { audiovisual } \\
\text { utilizado }\end{array}$ \\
\hline $1^{*}$ & Harvard & $\begin{array}{c}\text { Data Science, R } \\
\text { Basics }\end{array}$ & 552.278 & 24 & $1 \mathrm{~h} 26 \mathrm{~m}$ & VC-VA \\
\hline 2 & IBM & $\begin{array}{l}\text { Python Basics for } \\
\text { Data Science }\end{array}$ & 247.857 & 21 & $1 \mathrm{~h} 51 \mathrm{~m}$ & VC-VA \\
\hline $3^{* *}$ & $\begin{array}{l}\text { University of } \\
\text { Queenland }\end{array}$ & $\begin{array}{l}\text { IELTS Academic test } \\
\text { preparation }\end{array}$ & 1.133 .797 & 79 & $3 \mathrm{~h} 11 \mathrm{~m}$ & SVC-VAR-D \\
\hline 4 & Harvard & $\begin{array}{l}\text { Rhetoric, The art of } \\
\text { persuasive writing } \\
\text { and public speaking }\end{array}$ & 338.965 & 90 & $24 \mathrm{~h} 22 \mathrm{~m}$ & ANVC-VAE \\
\hline 5 & ETS & $\begin{array}{l}\text { TOEFL test } \\
\text { preparation, The } \\
\text { insider guide }\end{array}$ & 1.054 .089 & 48 & $1 \mathrm{~h} 51 \mathrm{~m}$ & SEVC-VAR-D \\
\hline
\end{tabular}

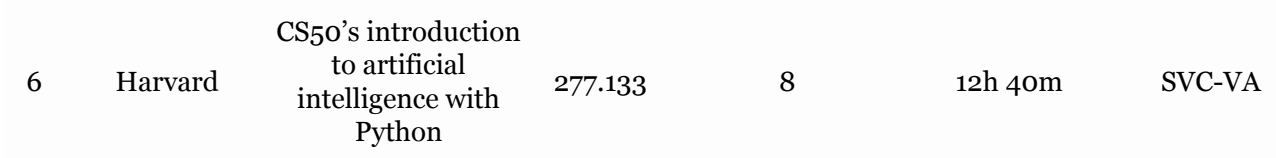




\begin{tabular}{ccccccc} 
No & $\begin{array}{c}\text { Universidad } \\
\text { o institución } \\
\text { formativa }\end{array}$ & Curso & $\begin{array}{c}\text { Número } \\
\text { total de } \\
\text { inscritos }\end{array}$ & $\begin{array}{c}\text { Total de } \\
\text { recursos } \\
\text { audiovisuales }\end{array}$ & $\begin{array}{c}\text { Total de } \\
\text { tiempo de } \\
\text { los recursos } \\
\text { audiovisuales }\end{array}$ & $\begin{array}{c}\text { Tipo de } \\
\text { material } \\
\text { audiovisual } \\
\text { utilizado }\end{array}$ \\
\hline 7 & Harvard & $\begin{array}{c}\text { Entrepreneurship in } \\
\text { emerging economies }\end{array}$ & 599.434 & 87 & $9 \mathrm{~h} 34 \mathrm{~m}$ & $\begin{array}{c}\text { VC- } \\
\text { VAANER-D }\end{array}$ \\
\hline 8 & Harvard & $\begin{array}{c}\text { Contract law, from } \\
\text { trust to promise to } \\
\text { contract }\end{array}$ & 423.559 & 81 & $6 \mathrm{~h} 12 \mathrm{~m}$ & VC-VA \\
\hline
\end{tabular}

Nota: *Se repite: está de primero en popularidad en “Análisis de Datos” y de segundo en "Informática"

**Se repite: está de primero en popularidad en "Humanidades" y en "Idiomas" Fuente: elaboración propia

En lo concerniente a los aspectos narrativos, destaca que de los ocho cursos todos recurren como método principal a la técnica Vídeoconferencia y Vídeoapunte, en la mayoría de los casos, emulando a la experiencia en un aula o un auditorio.

Caso más llamativo aún el del caso n. $^{\circ} 4$ "Rhetoric, The art of persuasive writing and public speaking", donde el profesor que lo imparte ha grabado el material directamente en el aula de clase. Diversas tomas con diferentes ángulos muestran al docente dando su clase habitual, donde interactúa con los alumnos allí presentes a los cuales insta a participar.

Son pocos los cursos de la muestra que recurren a las entrevistas como recursos didácticos. Los casos 4, 5 y 7 (Tabla 1) representan una alternativa de muy poco peso, siendo llamativo en el n. ${ }^{\circ}$, que, de noventa vídeos, solo siete fueron entrevistas (el $7,7 \%$ del material audiovisual).

Lo mismo ocurre con las animaciones, la cual en el curso $n .^{0} 4$ se limita a un solo vídeo pero que en el n. ${ }^{\circ} 8$ se representa un recurso inserto en la mayoría de las Vídeoconferencias, lo cual puede hacer eventualmente menos monótona la propuesta.

Cuando se señala en los cursos $n .^{0} 3,5$ y 7 contenidos con categoría de Reportaje y/o Documental (R-D), son solo vídeos muy concretos que buscan ilustrar o ampliar lo dicho en una vídeoconferencia determinada, pero no representa un soporte habitual. Convencionalmente no pasa de uno o dos en todo el programa.

Las dramatizaciones tampoco son un recurso explotado en los casos estudiados. Únicamente ha sido identificado en el n. ${ }^{\circ} 3$ (Universidad de Queenland) cuatro veces, representando apenas el 5,06\% de los recursos audiovisuales allí presentados.

El caso referido de los spots (n. ${ }^{0} 5$ y 6 , Tabla 1 ) son breves introducciones al curso, donde se hace la presentación de los docentes que intervendrán en él, y en el caso específico del n. ${ }^{\circ} 3$ (Universidad de Queenland) aprovechan para mostrar parte de las instalaciones de dicho centro educativo.

La mayor parte de los vídeos son del tipo videoconferencia o videoapunte, por tanto, las cuestiones narrativas se resumen en que básicamente, trasladan el 
aula al espacio online, a diferencia de que puedan añadir algún tipo de grafismo o animación, recurso que también se pueden incluir en una clase presencial del tipo clase magistral.

Todas las tipologías de vídeos que no están dentro de los formatos anteriormente señalados, como el spot (pieza audiovisual a modo de presentación del MOOC, incluso para la promoción del mismo), los reportajes y/o documental y las dramatizaciones, se sitúan en el modelo narrativo televisivo, es decir, transforman los contenidos educativos en formato televisivo, incluso los formatos de animación, pues en el espacio analógico, con otras posibilidades, también era posible la animación.

No se aprecia ninguna diferencia significativa en relación a las modelos que desarrolló la televisión educativa al trasladar los contenidos educativos al espacio audiovisual: reproducir en el espacio escolar presencial o reproducir el formato televisivo aprovechando todas las posibilidades expresivas del propio medio. El análisis de los datos obtenidos plantea resultados en esa dirección.

Obtenidos los resultados, con el fin de reforzar el análisis, bien vale establecer por igual una comparativa con respecto a los modelos educativos según el aporte de Yousef et al. (2014), quienes propusieron una clasificación en cuanto a tipos de MOOC y modelos de aprendizaje.

\section{Figura 1}

Tipos de MOOC

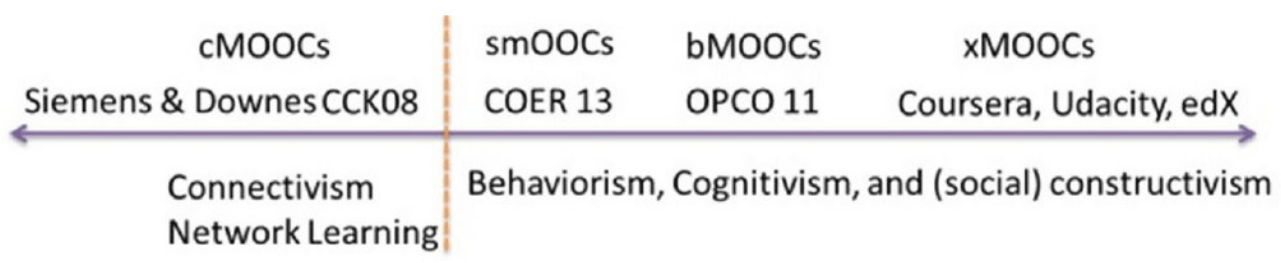

Fuente: Yousef et al. (2014)

Este esquema parece complementar la respuesta al tipo de material audiovisual del que se hace uso en estos MOOC. Se parte de la premisa que el trabajo volcado en las plataformas como edX pertenecen al grupo de los XMOOx, lo que indica que están dentro del modelo constructivista.

\section{DISCUSIÓN Y CONCLUSIONES}

El que un contenido educativo se ubique dentro del espectro del modelo constructivista pone el acento en la interacción profesor-estudiante de esfuerzo por parte del estudiante por apropiarse del conocimiento, al tiempo que se desarrolla dentro de una estructura más focalizada, distando así del modelo educativo 
conectivista desarrollado por Siemens y Downes (Torres y Gago, 2014, p. 14) y, así mismo, sus contenidos y, por tanto, el material audiovisual, suponiendo por tanto una respuesta al por qué es posible encontrarse reiteradamente con tipologías de vídeos como las videoconferencias o videoapuntes.

Ante los resultados obtenidos ¿̇cuáles son los retos frente a un contexto online para la educación en el que se abren muy diversas formas de modelos aprendizaje y posibilidades para el mismo, pero en el que triunfan, principalmente, los modelos de enseñanza más tradicionales?

Se afirma que una investigación denominada como tal, tiene su sentido en avanzar sobre lo investigado o en restructurar o replantear las premisas prestablecidas, sobre las que se parte. Siendo esto así, se puede afirmar que el proceso de elaboración del presente artículo ha seguido esos caminos, pues en el proceso de estudio de la temática en cuestión, las premisas preestablecidas, los interrogantes de partida y las búsquedas de partida, han planteado nuevos interrogantes y, por tanto, ha llevado a nuevos aprendizajes y a la posibilidad de poder señalar nuevos retos y propuestas.

Se partió del supuesto de que los MOOC presentaban nuevas metodologías y narrativas para el aprendizaje y, como posibilidad, sin duda, es un hecho y tiene sentido pensarlo así, en una realidad de código binario.

Ya hace unos años García-Peñalvo et al. (2017) planteaban la necesidad de evolucionar en el modelo de los MOOC con el fin de reforzar la transmisión del conocimiento desde las instituciones educativas, así como procurar nuevos espacios de diálogo entre profesores y alumnos.

Pero, precisamente, la realidad tangible es que 3 años después de eso, queda en evidencia que se repiten modelos y formas de aprendizaje tradicionales siendo, además, como ya se ha señalado, los que resultan más demandados.

En coincidencia con Rajas et al. (2018), inclusive estos contenidos carecen en la mayoría de los casos, de una realización audiovisual destacada. Sin embargo, donde se puede decir que reside la aportación significativa de estos formatos, está en llevar el conocimiento, el aprendizaje a cualquier lugar o, mejor aún, a aquellos lugares que de otro modo no llegaría, la misma que la de la Televisión Educativa.

No obstante, en el contexto digital se da la posibilidad de una vuelta de tuerca más, su capacidad de conectividad entre todos eso lugares del mundo que se hacen partícipes de compartir unos mismos contenidos, la capacidad de crear una red de aprendizaje, una comunidad de aprendizaje infinita, la posibilidad de hacer real, tangible, lo que se ha dado en llamar inteligencias colectivas. Ahí es donde se debe poner el principal foco de interés, de su verdadera y genuina potencialidad. El poder poner en red a los mayores especialistas en determinadas materias y el poder estar en conexión con ellos, generando nuevas conexiones.

Respecto al material audiovisual, se entiende que éste juega un papel fundamental en ello pues, de primeras, ofrece un valor especialmente significativo que, más allá de si éste es una videoconferencia o una dramatización, te permite "asistir" a clases de los mayores expertos de una materia determinada y ponerte en red, en conexión a 
todos los participantes que comparten un mismo interés, independientemente de su grado de especialización en la materia dada.

Este es un aporte significativo por parte del material audiovisual, pero es aún mayor si se suman todos los recursos expresivos del lenguaje audiovisual, los grafismos, las animaciones. Y qué se puede decir respecto a la interacción.

Evidentemente, como primera evidencia, el vídeo no permite interacción con el docente, el instructor, como se denomina en otros foros, a no ser que sea en streaming, pero tampoco sería posible en un contexto de miles de matriculados.

El vídeo puede ser visualizado en numerosas ocasiones, todas las que sean necesarias, pero sin poder interactuar en sí, lo cual pierde el componente dialógico, fundamental en todo proceso de aprendizaje, si se toman en consideración las apreciaciones de Aparici (2016) referidas en la introducción, que colocan en el centro del aprendizaje la participación, que se traduce en una interacción definida por el diálogo entre todos los implicados en el proceso de aprendizaje, profesores/ as y alumnos/as.

YouTube permite crear vídeos interactivos con preguntas y enlaces web, diseñando diferentes rutas de aprendizaje, pero en los materiales analizados no se hace uso de esta posibilidad, por ejemplo.

Más allá de los espacios interactivos que se ofrezcan, de los espacios para compartir conocimiento (foros, chat, etc.), desde una posición propositiva, se podrían y deberían plantear materiales audiovisuales que aprovecharan más los recursos expresivos audiovisuales, así como la inclusión de videos interactivos como los señalados.

Frente a los cMOOC, éstos, en principio, se puede decir que sí ofrecen un mayor grado de interactividad pues sus cursos se conforman no en una plataforma definida, sino entre muy diversos medios y/o plataformas de forma colaborativa, como establece por igual Ruiz Bolívar (2015, p. 11).

A partir de la paradoja de interactividad que plantean estos formatos, quizás esté la explicación de que eventualmente sean menos reclamados o con una baja tasa de matriculados. Vale establecer una comparativa con los formatos $360^{\circ}$ para entenderlo mejor: en los formatos $360^{\circ}$ el espectador puede, en principio, elegir el encuadre, pero esta elección de encuadre se hace con base a unas premisas y diseños previos del director, es la paradoja de guiar la libertad de interactividad del usuario.

En el momento en el que al espectador se le da la posibilidad de elegir el encuadre, redefine la interactividad con el contenido ya que se le otorga el rol de protagonista, lo cual redefine la acción de ver como una experiencia más cercana que intenta involucrarlo.

El objetivo máximo de estos modelos de aprendizaje debe ser ese, pero resulta imperativo superar la paradoja de la libertad de interacción del usuario, ya que la clave de la baja tasa de éxito de este formato, en coincidencia con las apreciaciones de Zapata-Ros (2015) recae en la casi ausencia de diseño instruccional de los MOOC desde sus principios. 
En ese sentido, es posible entender que este grado de interactividad puede ser significativo si se parte de unos participantes con grado de especialización significativo en la materia, así como con un alto grado de motivación e implicación. Y, por otro lado, si se consigue superar, tanto por parte del alumnado como del profesor, las resistencias a estos modelos cuando por ambas partes se procede de esquemas tradicionales de aprendizaje, es decir, en relación a la comparativa establecida, la misma sensación de desorientación que pueden experimentar tanto los directores de cine como espectadores cuando el primero se ve en la tesitura que debe prescindir del encuadre (se dispone de una única panorámica $360^{\circ}$ ) y el espectador elegirlo.

Ante ello los tMOOC se vislumbran como la respuesta idónea pues combinan ambos formatos, pero también, resulta necesario superar los planteamientos interiorizados de la formación tradicional en un contexto red que ofrece y abre otras posibilidades. Se aprovecha su potencial en red, pero a partir de todas las posibilidades que sus narrativas ofrecen, tanto para experimentar otras vías de aprendizaje, otras metodologías, a la par que retroalimentar las ya existentes, enriquecer éstas, así como la experiencia de la enseñanza presencial.

\section{NOTAS}

1. Concepto definido por la Carta Europea para la Alfabetización en los Medios de Comunicación y que se lanza de manera formal el 28 de septiembre del 2006, cuyo espacio fue la web que se lanzó en la primavera del mismo. https://euromedialiteracy. eu

2. «Declaración de Grünwald en Educación en Medios» (1982), Coloquio de Toulouse «Nuevas direcciones en Educación en Medios» (1990), Conferencia de Viena, «Educando para la era digital». (1999). Pérez Tornero (2007, p. 129). La estrategia internacional de la educación en medios. https://recyt.fecyt.es/index.php/comunicar/ article/view/25986

\section{REFERENCIAS}

Andretta, S. (2009). Transliteracy: take a walk on the wild side. Proceeding World Library and Information Congress: 75th IFLA General Conference and Council, 23-27. Milán, Italia. https://bit. ly/3sDQiG8

Aparici, R. (2016). Educomunicación y voces de la educación mediática. Una propuesta de Roberto Aparici. Entrevista a Daniel Prieto Castillo, educador, Universidad Nacional de Cuyo, Mendoza (Argentina). Iván Rodríguez Cuevas (realizador-redactor
CEMAV, UNED). https://canal.uned.es/ video/5a6f239gb1111fo23f8b456a

Barbas Coslado,A.(2012). Educomunicación: desarrollo, enfoques y desafíos en un mundo interconectado. Foro de Educación, 14, 157-175.

Baysal, S. (2014). Working with generations $\mathrm{X}$ and $\mathrm{Y}$ in generation $\mathrm{Z}$ period: management of different generations in business life. Mediterranean Journal of Social Sciences, 5(19). 218-229. http:// doi.org/10.5901/mjss.2014.v5n19p218 
Clark, D. (2013). MOOCs: taxonomy of 8 types of MOOC. Blogger. http://bit. ly/3nZZOQl

Conole, G. (2013). MOOCs as disruptive technologies: strategies for enhancing the learner experience and quality of MOOCs. Revista de Educación a Distancia, RED, 39. $\quad$ https://www.um.es/ead/red/39/ conole.pdf

Freire, P. (1968). Pedagogía del oprimido. Siglo XXI.

García-Peñalvo, F. J., Fidalgo-Blanco, Á., y Sein-Echaluce, M. L. (2017). Los MOOC: un análisis desde una perspectiva de la innovación institucional universitaria. $L a$ Custión Universitaria, 9, 117-135.

Gil-Quintana, J., y Osuna-Acedo, S. (2020). Transmedia Practices and Collaborative Strategies in Informal Learning of Adolescents. Social Sciences, 9(6), 92. https://doi.org/10.3390/socscigo60092

Ipri, T. (2010). Introducing transliteracy. What does it mean to academic libraries? November College \& Research Libraries News, 71(10), 532-567 https://doi. org/10.5860/crln.71.10.8455

Kaplún, M. (1998). Una pedagogía de la comunicación. Ediciones de la Torre.

King, M., Pegrum, M., y Forsey, M. (2018). MOOCs and OER in the Global South: problems and potential. International Review of Research in Open and Distributed Learning, 19(5), 1-20. https:// doi.org/10.19173/irrodl.v19i5.3742

Levy, P. (2004). Inteligencia colectiva. Por una antropología del ciberespacio. Washington: Organización Panamericana de la Salud.

Makazaga, I. (2020). La radio, aliada contra la pandemia en África. Planeta Futuro. El País http://bit.ly/3npz4cm

OECD (2018). G2O Leaders' declaration. Building consensus for fair and sustainable development. Education Policy Outlook 2018. http://bit. ly/2XSaFBp

Osuna-Acedo, S., Marta-Lazo, C., y FrauMeigs, D. (2018). De sMOOC a tMOOC, el aprendizaje hacia la transferencia profesional: El proyecto europeo ECO. Comunicar, 55, 105-114. https://doi. org/10.3916/C55-2018-10

Osuna-Acedo, S. (2014). Escenarios virtuales educomunicativos. Icaria Editorial.

Pérez Tornero, J. M. (2007). La estrategia internacional de la educación en medios. Comunicar, 28, 129-132.

Prieto Castillo, D. (2004). Presencia de la comunicación educativa. En R. Aparici (ed.) Comunicación educativa en la sociedad de la información. UNED.

Rajas, M., Puebla-Martínez, B., y Baños, M. (2018). Formatos audiovisuales emergentes para MOOCS: diseño informativo, educativo y publicitario. $E l$ profesional de la información, 27(2), 1699-2407. https://doi.org/10.3145/ epi.2018.mar.09

Rheingold, H. (2004). Multitudes inteligentes. La próxima revolución social. Gedisa.

Ruiz Bolívar, C. (2015). El MOOC: dun modelo alternativo para la educación universitaria? Revista Apertura, 7(2). 1-14.

Scolari, C. (2008). Hipermediaciones. Elementos para una teoría de la comunicación digital interactiva. Gedisa.

Suárez, C. (2010). La formación en red como objeto de estudio. Revista de Universidad y Sociedad del Conocimiento (RUSC), $7(2)$.

Sukovic, S. (2016). Transliteracy in Complex Information Environments. Chandos Publishing. https://doi.org/10.1016/ B978-0-08-100875-1.00004-2

Toffler, A. (1980). The third wave. Bantam Books

Torres Mancera, D., y Gago Saldaña, D. (2014). Los MOOCs y su papel en la creación de comunidades de aprendizaje y participación. RIED. Revista Iberoamericana de Educación a Distancia, 17(1), 13-34. https://doi. org/10.5944/ried.17.1.11570 
Turner, A. (2015). Generation Z: technology and social interest. The Journal of individual psychology, 71(2). 103-113. https://doi.org/10.1353/jip.2015.0021

UNESCO (2011). Alfabetización mediática $e$ informacional: curriculum para profesores. https://bit.ly/2K8HcQf

Wilkinson, L. (2011). Transliteracy or Metaliteracy? Libraries and Transliteracy. $\quad$ https:// librariesandtransliteracy.wordpress. com/2011/02/01/transliteracy-ormetaliteracy/
Yousef, A. M. F., Chatti, M. A., Schroeder, U., Wosnitza M., y Jakobs, H. (2014). MOOCs - A Review of the State-of-theArt. Proceedings of 6th International Conference on Computer Supported Education. CSEDU 2014 conference, 3, 9-20.

Zapata-Ros, M. (2015). El diseño instruccional de los MOOC y el de los nuevos cursos abiertos personalizados. RED-Revista de Educación a Distancia. 45(2), 1-35. https://doi.org/10.6018/ red/45/zapata

\section{PERFIL ACADÉMICO Y PROFESIONAL DE LAS AUTORAS}

Raquel Caerols Mateo. Doctora en Creatividad Aplicada. Líneas de investigación en arte, nuevos medios y creatividad, desarrollando actualmente estas líneas de trabajo en el contexto de los medialab, como espacios destacados para la alfabetización mediática, lo que le ha llevado a liderar, junto con el profesor Isidro Moreno, el proyecto: MediaLab Madrid como modelo de laboratorio transversal: arte, ciencia, tecnología, sociedad + sostenibilidad para la agenda digital (H2019/ HUM-5740 (MediaLab-CM).

E-mail: rcaerols@ucm.es

\section{DIRECCIÓN DE LA AUTORA}

Facultad Ciencias de la Información

Universidad Complutense de Madrid

Avda. Complutense, 3.

28040 Madrid (España)

Pavel Sidorenko Bautista. Doctor por la Facultad de Periodismo de la Universidad de Castilla-La Mancha. Es profesor en la Facultad de Ciencias de la Comunicación de la Universidad Francisco de Vitoria (Madrid, España) y del Máster en Comunicación e Identidad Corporativa de la Universidad Internacional de La Rioja (España). Ha sido profesor en la Universidad de Castilla-La Mancha, Universidad Central de Venezuela y en la Universidad Monteávila (Caracas, Venezuela). Centra su investigación en el desarrollo y aplicación de nuevas narrativas y tecnologías en diferentes ámbitos de la Comunicación: periodismo, publicidad, marketing y comunicación corporativa. Investigaciones y otros escritos pueden consultarse en https://bit.ly/PavelSidorenkoBautista

E-mail: pavel.sidorenko@ufv.es 


\section{DIRECCIÓN DEL AUTOR}

Facultad de Ciencias de la Comunicación

Universidad Francisco de Vitoria

Ctra. Pozuelo-Majadahonda, KM. 1.800

28223 Pozuelo de Alarcón, Madrid (España)

Sara Osuna-Acedo. Doctora en Filosofía y Ciencias de la Educación por la UNED desde el año 2004. Es profesora de Comunicación y Educación en la UNED. Sus líneas de investigación son MOOC (sMOOC y tMOOC), convergencia de medios, escenarios digitales, discapacidad y diseño para todos, aprendizaje digital y redes sociales, publicando artículos científicos en revistas internacionales de primer nivel. E-mail: sosuna@edu.uned.es

\section{DIRECCIÓN DE LA AUTORA}

UNED

Facultad de Educación

Calle Juan del Rosal, 14; $2^{\circ}$, despacho 236

28040 Madrid

Fecha de recepción del artículo: 16/02/2021

Fecha de aceptación del artículo: 06/07/2021

Fecha de aprobación para maquetación: 02/08/2021 